\title{
Propagators in Yang-Mills theory for different gauges
}

\section{Axel Maas*}

E-mail: axelmaas@web.de

\section{Attilio Cucchieri}

E-mail: attilio@ifsc.usp.br

\section{Tereza Mendes}

E-mail: mendes@ifsc.usp.br

Instituto de Física de São Carlos, Universidade de São Paulo, Caixa Postal 369, 13560-970 São Carlos, SP, Brazil

Green's functions are gauge-dependent quantities. Thus, the manifestation of confinement in these correlation functions also depends on the gauge. Here we use lattice gauge theory to study the gluon and the ghost propagators in a gauge (the so-called $\lambda$-gauge) interpolating between the Landau and the Coulomb gauge. Results are compared to the usual minimal Landau gauge.

8th Conference Quark Confinement and the Hadron Spectrum September 1-62008

Mainz, Germany

${ }^{*}$ Speaker. 
Green's functions encode completely the non-perturbative properties of a quantum field theory. Thus, the infrared (IR) behavior of the gluon propagator $D(p)$ in Yang-Mills theory should contain information on confinement. In particular, a violation of reflection positivity (at large separation) for the gluon propagator is considered an unambiguous signal for the confinement of gluons [1]. This issue has been widely studied in Landau gauge. Continuum methods, such as Dyson-Schwinger equations find such a behavior for the gluon propagator [1]. A clear sign of violation of reflection positivity for the gluon is also obtained using lattice numerical simulations in the $3 \mathrm{~d}$ case [2] while, in $4 \mathrm{~d}$, finite-size effects and technical limitations have not allowed yet a clear verification of this result [3]. At the same time, the Landau ghost propagator shows a divergent behavior [1,3], stronger than $p^{-2}$ for small momenta $p$. This strong IR divergence corresponds to a long-range interaction in real space, which may be related to quark confinement. On the other hand, in order to understand the confinement mechanism, it is important to verify how these IR features are modified when considering other gauges [4]. Thus, it is important to investigate these correlation functions in a wide class of gauges. Particularly important are gauges depending on a parameter, since one can interpolate between different gauge conditions. This allows one to investigate changes in the correlations functions while varying smoothly the gauge parameter (i.e. the gauge condition).

Let us recall that the gauge-fixed Lagrangian density in the (Euclidean) continuum can be written as

$$
\mathscr{L}=\frac{1}{4 g^{2}} F_{\mu \nu}^{a} F_{\mu \nu}^{a}+\frac{1}{2 \xi} \partial_{\mu}^{\prime} A_{\mu}^{a} \partial_{v}^{\prime} A_{v}^{a}+\bar{c}^{a} \partial_{\mu}^{\prime} D_{\mu}^{a b} c^{b}
$$

Here, $\partial_{\mu}^{\prime}=\lambda \partial_{0}+\partial_{i}, A_{\mu}^{a}$ is the gauge field, $D_{\mu}^{a b}$ is the usual adjoint covariant derivative, $F_{\mu \nu}^{a}$ is the field-strength tensor and the ghost fields $\bar{c}^{a}$ and $c^{a}$ have been introduced in order to exponentiate the Jacobian, i.e. the Faddeev-Popov operator $\partial_{\mu}^{\prime} D_{\mu}^{a b}$, introduced by fixing the gauge. Note that, for $\lambda=1$, the quadratic term in $\partial_{\mu}^{\prime} A_{\mu}^{a}$ corresponds to the usual perturbative definition of linear covariant gauges with gauge parameter $\xi$. In particular, $\xi=1$ is the Feynman gauge and the limit $\xi=0$ corresponds to Landau gauge. On the other hand, for $\xi=0$, the parameter $\lambda$ allows to interpolate between the Landau gauge $(\lambda=1)$ and a Coulomb-like gauge $(\lambda=0)$. Note that the usual Coulomb gauge $\partial_{i} A_{i}^{a}=0$ has to be supplemented by further constraints [5] to yield the limit $\lambda=0$.

Here, we present results for the case $\xi=0$ (the so-called $\lambda$-gauge) obtained using lattice numerical simulations for the $S U(2)$ gauge group. To be able to reach the IR asymptotic regime, we consider the $3 \mathrm{~d}$ case, extending earlier studies of this subject [6]. Clearly, for $\lambda<1$, Euclidean invariance is explicitly broken by the gauge condition. In this case, there are two independent kinematic variables, namely $p_{0}$ and $|\vec{p}|$. At the same time, the gluon propagator $D_{\mu v}\left(p_{0},|\vec{p}|\right)$ also has two independent tensor structures: its temporal component $D_{00}\left(p_{0},|\vec{p}|\right)$ and its spatial projection $D^{\operatorname{tr}}\left(p_{0},|\vec{p}|\right)$. Predictions exist [5] that both components of the gluon propagator vanish at zero total momentum, $p_{0}^{2}+|\vec{p}|^{2}=0$.

In Figure 1 we report results for $D^{\operatorname{tr}}\left(p_{0},|\vec{p}|\right)$ as a separate function of $p_{0}$ and of $|\vec{p}|$ for different values of $\lambda$. We find that, as a function of $|\vec{p}|$, the maximum of $D^{\operatorname{tr}}$ moves to larger momenta as $\lambda$ decreases. On the contrary, when considering its dependence on $p_{0}$, we see that the maximum of $D^{\text {tr }}$ moves to smaller momenta and that, already for $\lambda=1 / 10$, the maximum can no longer 

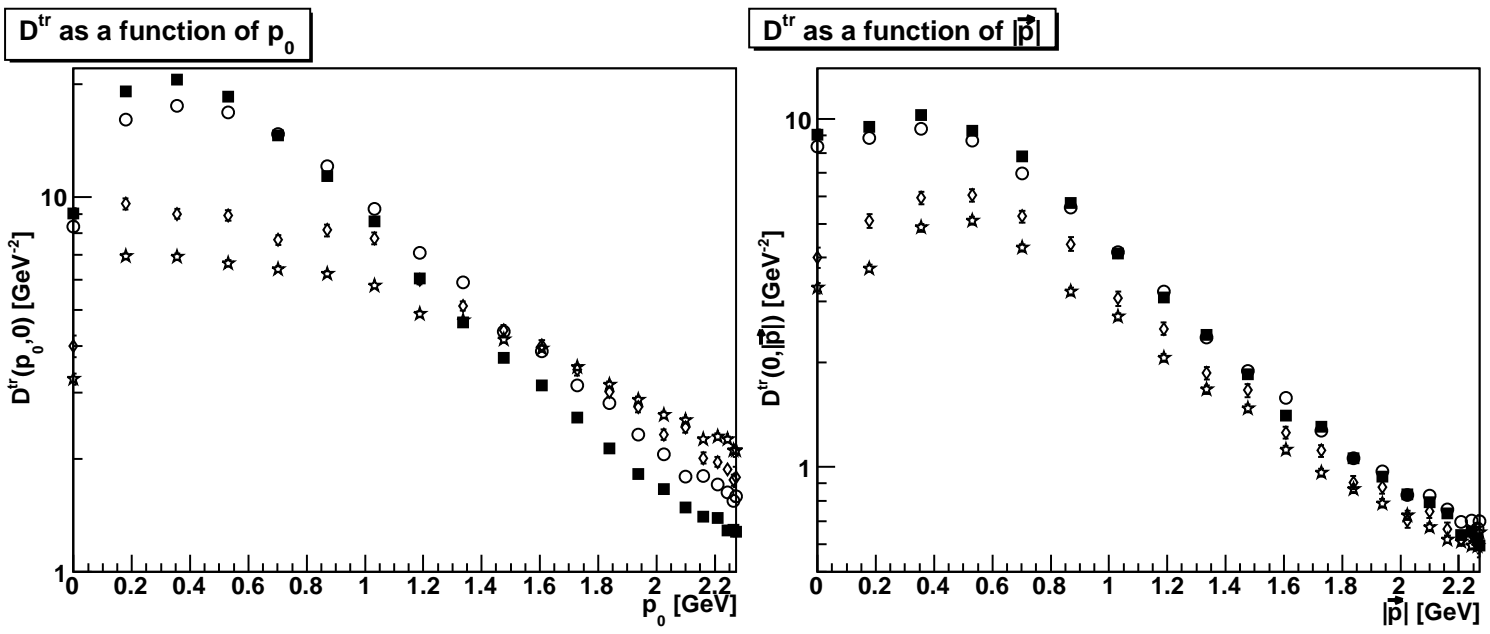

Figure 1: The gluon propagator $D^{\operatorname{tr}}\left(p_{0},|\vec{p}|\right)$ as a function of $\lambda$ for the lattice volume $40^{3}$ at $\beta=4.2$. In the left panel we report data for $D^{\operatorname{tr}}\left(p_{0}, 0\right)$ while in the right panel we consider $D^{\operatorname{tr}}(0,|\vec{p}|)$. We represent $\lambda=1$ with black squares, $\lambda=1 / 2$ with open circles, $\lambda=1 / 10$ with diamonds and $\lambda=1 / 20$ with stars.
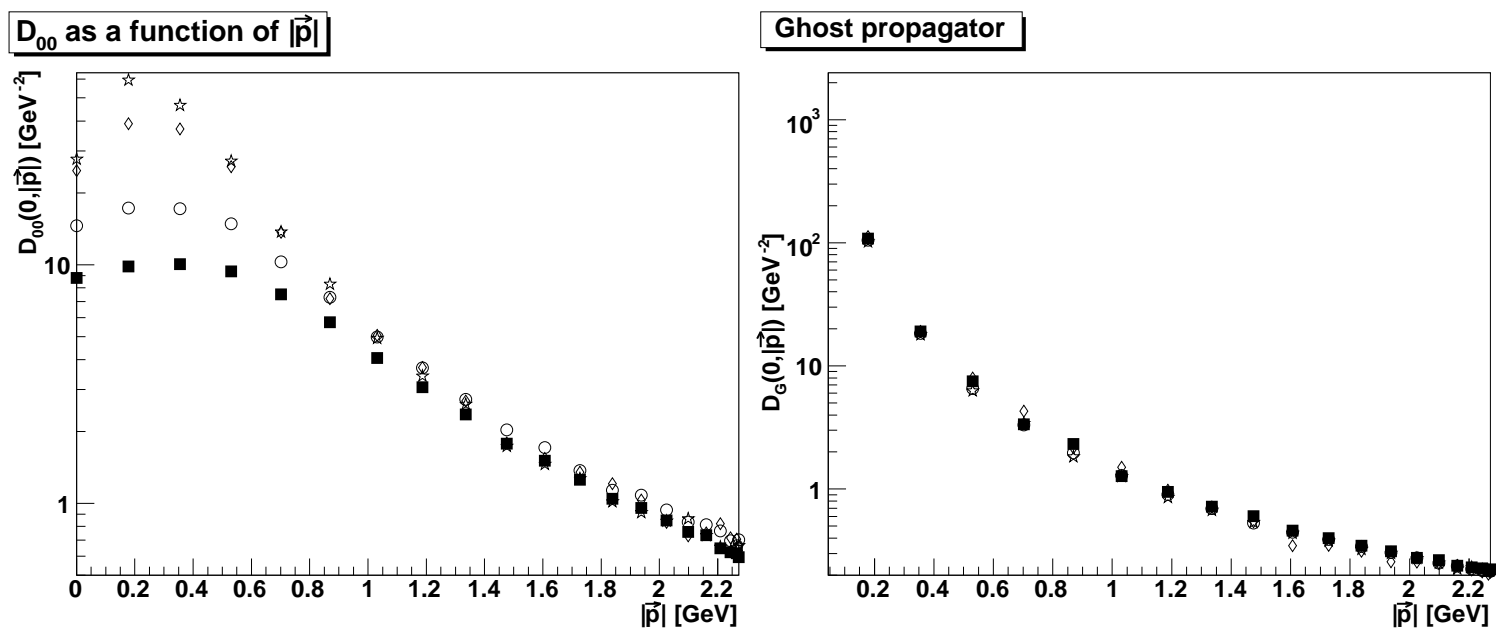

Figure 2: In the left panel we show results for the gluon propagator $D_{00}(0,|\vec{p}|)$, while in the right panel we report data for the ghost propagator $D_{G}(0,|\vec{p}|)$. In both cases we consider various values of $\lambda$ (symbols and lattice as in Figure 1). Note that the quantity $D_{00}\left(p_{0}, 0\right)$ vanishes identically due to the gauge condition.

be resolved. In both cases, for small momenta, the propagator decreases as $\lambda$ gets smaller. The function $D_{00}(0,|\vec{p}|)$ is considered in the left panel of Figure 2. In this case, as $\lambda$ decreases, the maximum of the propagator moves to smaller momenta while the propagator increases in the IR. This is somewhat expected, since in Coulomb gauge this tensor component diverges [7]. Thus, as $\lambda$ decreases, the gluon propagator exhibits more and more a Coulomb-like behavior. Finally, the $\lambda$ dependence for the ghost propagator is shown in the right panel of Figure 2. We see that, as a function of $|\vec{p}|$, it is essentially independent of $\lambda$. On the other hand, when $D_{G}\left(p_{0},|\vec{p}|\right)$ is considered as a function of $p_{0}$ we find that it increases approximately linearly with $\lambda$ [8]. These results support the findings of recent DSE studies [5]. A more extensive numerical study for this 
interpolating gauge will be presented elsewhere [8].

Finally, let us note that the study of the linear covariant gauges on the lattice is much more involved. In particular, at $\xi>0$ and $\lambda=1$, the continuum gauge condition $\partial A_{\mu}^{a}(x)=\Lambda^{a}(x)$ cannot be implemented for any finite $\beta$ because $\Lambda^{a}(x)$ has a Gaussian distribution, while the values of $\partial A_{\mu}^{a}(x)$ are clearly bounded. Results for the linear covariant gauges will be presented elsewhere [9].

Acknowledgements A. M. is grateful to the organizers for the opportunity to present this work. A. M. was supported by the DFG under grant numbers MA 3935/1-1 and MA 3935/2-1. A. C. and T. M. were supported by FAPESP (under grants \# 00/05047-5 and 05/59919-7) and by CNPq.

\section{References}

[1] R. Alkofer and L. von Smekal, Phys. Rept. 353, 281 (2001).

[2] A. Cucchieri, T. Mendes and A. R. Taurines, Phys. Rev. D 71, 051902 (2005).

[3] A. Cucchieri and T. Mendes, Braz. J. Phys. 37 (2007) 484 [arXiv:hep-ph/0605224].

[4] See for example studies in Coulomb gauge (A. Cucchieri, AIP Conf. Proc. 892 (2007) 22 [arXiv:hep-lat/0612004]) or in maximally Abelian gauge (T. Mendes, A. Cucchieri and A. Mihara, AIP Conf. Proc. 892 (2007) 203 [arXiv:hep-lat/0611002].).

[5] C. S. Fischer and D. Zwanziger, Phys. Rev. D 72, 054005 (2005).

[6] A. Cucchieri, F. Karsch and P. Petreczky, Phys. Rev. D 64, 036001 (2001).

[7] A. Cucchieri and D. Zwanziger, Phys. Rev. D 65, 014001 (2002).

[8] A. Cucchieri, A. Maas and T. Mendes, Mod. Phys. Lett. A 22 (2007) 2429 [arXiv:hep-lat/0701011]; A. Cucchieri, A. Maas, T. Mendes, in preparation.

[9] A. Cucchieri, A. Maas, T. Mendes, arXiv:0806.3124 [hep-lat], accepted by CPC. 